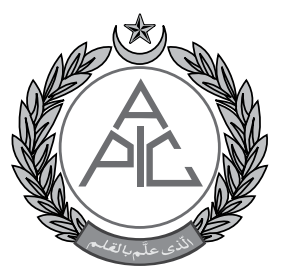

\title{
The role of neuromuscular electrical stimulation for rehabilitation in ICU acquired weakness
}

\author{
Martha Kurnia Kusumawardani ${ }^{1}$, Dewi Poerwandari ${ }^{1}$, \\ Inggar Narasinta ${ }^{1}$, Hanik Badriyah Hidayati ${ }^{2}$, Imam Subadi ${ }^{1}$
}

\begin{abstract}
Objective: To provide an overview of the muscle weakness development in intensive care units (ICU), summarize clinical trials on the role of neuromuscular electrical stimulation for muscle weakness rehabilitation in ICU and highlight recent strategies that may prevent or minimize this condition.
\end{abstract}

'Department of Physical Medicine and Rehabilitation; 2Department of Neurology, Faculty of Medicine, Airlangga University / Dr. Soetomo General Hospital, Surabaya, East Java, Indonesia

\section{Correspondence:}

Hanik Badriyah Hidayati, Department of Neurology, Faculty of Medicine, Airlangga University / Dr. Soetomo General Hospital, Surabaya, East Java, Indonesia; E-mail: hanikhidayati@yahoo.com

Received: 25 Oct 2018

Reviewed: 4 Nov 2108

Accepted: 8 Nov 2108

\section{Methodology: Literature review.}

Results: Some recent studies have shown that an increasing number of survivors of critical illness develop significant functional impairment; one of the common impairments being ICU acquired muscle weakness. Prolonged immobilization and mechanical ventilation likely contribute to the development of ICU-acquired weakness. Several novel therapeutic strategies have been used to overcome the impairment, including neuromuscular electrical stimulation. This neuromuscular electrical stimulation, a kind of physical therapy, is known to stimulate the nerves and thus increase muscle contraction.

Conclusion: Neuromuscular electrical stimulation for rehabilitation in ICU-related muscle weakness has shown some promise, but more efforts are needed to detect the development of ICU-acquired weakness as early as possible and rigorously evaluate novel rehabilitation interventions.

Key words: Critical illness; Muscle strength; Post-ICU Rehabilitation; Neuromuscular Electrical Stimulation, Physiotherapy

Abbreviations: ICU (Intensive Care Unit); ICU-Acquired Weakness (ICU-AW); CIP (Critical Illness Polyneuropathy); CIM (Critical IIIness Myopathy), CIPM (Critical Illness Polyneuropathy and Myopathy)

Citation: Kusumawardani MK, Poerwandari D, Narasinta I, Hidayati HB, Subadi I. The role of neuromuscular electrical stimulation for rehabilitation in ICU acquired weakness. Anaesth Pain \& Intensive Care 2018;22 Suppl 1:S164-S169

\section{BACKGROUND}

Life expectancy of patients with critical illness has been continuously increasing over decades due to rapid development of newer diagnostic modalities, newer therapeutic agents, improved clinical and nursing care in these patients. This condition inevitably lead to an increasing numbers of prolonged immobilization patients in intensive care units (ICU). Prolonged immobilization my adversely affect the organ function, resulting into significant functional impairment and a poor quality of life..$^{1,2}$ Generallized muscular weakness without any exact causes during treatment in the ICU, usually called ICU-Acquired Weakness (ICU-AW), is one of the common functional impairments in patients with critical illness. It may result into prolonged mechanical ventilation, delayed recovery, and thus prolonged ICU stay, significantly increasing the risk of morbidity and mortality. ${ }^{3-5}$ 
Prolonged ICU stay causes a decrease in muscle activity resulting in muscle atrophy and a decrease in muscle strength. Muscles that develop atrophy also have a reduced number of capillaries in muscle fibers. The arterial walls become thickened and hardened, reducing the capacity to dilate. ${ }^{2}$

The etiology of ICU-AW is multifactorial, yet prolonged immobilization remains the most common deconditioning factor. Early diagnosis of muscle weakness is important to plan for early ambulation, to prevent the failure of weaning and predict outcomes. ${ }^{3,4,6}$ ICU-AW is one of the complications that often arises from critical illnesses and is associated with high rates of morbidity and mortality. The latest data shows that the incidence of ICU-AW has long term consequences, beyond the time of hospitalization. ICU-AW is very likely to be an important contributor to post intensive care syndrome. This terminology includes persistent physical, mental and cognitive dysfunction. ${ }^{7}$ There may be involvement of the peripheral and central nervous system. Several terms have been used for this group of disorders, including critical illness polyneuropathy (CIP), critical illness myopathy (CIM), and critical illness polyneuropathy and myopathy (CIPM). ${ }^{8}$

\section{PATHOPHYSIOLOGY}

There are several risk factors that influence the occurrence of ICU-AW, including prolonged immobilization, systemic inflammation and over sedation. There are several risk factors that are still considered controversial by many researchers, including the use of corticosteroids, neuromuscular blocking agents and hyperglycemia. ${ }^{4}$

Although some studies have shown several pathological conditions that might underlie the onset of ICU-AW, the exact cause remains unclear,' hence the pathophysiological mechanism of ICU$\mathrm{AW}$ is believed to be multifactorial, including the microcirculation dysfunction and hyperglycemia. The former may cause nerve ischemia that lead to axonal degeneration. Inactivation of sodium channels was also thought to be a trigger of neuropathy, myopathy or both of them in critical illness. ${ }^{10}$

\section{DIAGNOSIS}

ICU-AW variations, such as CIP, CIM, and CIPM, have similar features and are difficult to distinguish clinically. This condition can exist beyond first week of ICU stay, in patients with systemic inflammation, multiple organ failure, administered with high doses of corticosteroids or neuromuscular blocking agents. 5

The diagnosis of CIP is made with the presence of all of the following:

1. Patient meets the criteria for ICU-AW

2. CMAP amplitudes are decreased to $<80 \%$ of the lower limit of normal in $>2$ nerves

3. SNAP amplitudes are decreased to $<80 \%$ of the lower limit of normal in $>2$ nerves

4. Normal or near normal nerve conduction velocities

5. Absent decremental response on repetitive nerve stimulation.

The CIM diagnostic criteria are the occurrence of 1 , 2, 5, and either 3 or 4 :

1. Muscle weakness that develops after the onset of critical illness

2. The weakness begins as generalized (starts at the proximal limb muscles, continues to distal limbs), symmetrical, flaccid and spares the cranial nerves.

3. The muscle strength assessed using Medical Research Council (MRC) score. A composite MRC score from the examination of 3 muscle groups in each limb (range: 0 to 5), with $<80 \%$ of the maximum score (ie, $<48$ out of 60 ).

4. Dependent mechanical ventilation.

5. Caused muscle weakness is not related to the underlying disease of the patient.

The earliest signs can be facial grimace without extremity movements in response to pain stimulation. It is rare to find extra ocular muscle involvement. The muscle atrophy is variable and often disguised as edema. In addition, a weakness in respiratory muscles can also appear and affect the weaning process of mechanical ventilation. ${ }^{4,5,10}$

Patients, who have been diagnosed as ICU-AW based on the criteria, should undergo a serial evaluation; and if the neurological deficit persists, further examination can be recommended in the form of electrophysiological studies and muscle biopsy. Meanwhile, in patients with persistent coma after cessation of sedation, CT-scan or MRI of central nervous system is recommended. ${ }^{10,11}$

\section{NEUROMUSCULAR ELECTRICAL STIMULATION (NMES)}

Difference in gradient concentration and voltage in our body induce electrical transmission of the cell and affect the depolarization. Electrical stimulation basically is a neurologic process. ${ }^{12,13}$

The body tissues such as muscles, nerves, body 
fluids are a conductor, so the electric current can pass through the tissue. On the other hand the skin, hair, ligaments, callus, fat, bone, tendon, and scar are insulators. ${ }^{13,14}$

Neuromuscular electrical stimulation (NMES) is an application of electrical stimulation to produce skeletal muscle contraction as a result of transcutaneous peripheral stimulation. The purpose of NMES application are for muscle strengthening especially after prolonged immobilization, maintain muscle mass, and selective muscle training. ${ }^{15}$

Commonly NMES is used for musculoskeletal rehabilitation, but beside that, it is also used for patients with central nervous system injury. It creates an electrical field around motor axon that have sufficient strength to make depolarization at the axonal membrane, so the action potential will occur and induces muscle contraction. Thus, NMES works by depolarizing motor axons rather than muscle fibers directly. To be effective, NMES application needs an intact peripheral nerve and healthy muscle physiology. It has a specific effect of improving function but does not directly result in function. ${ }^{16}$ It is an electrical stimulation used for an intact lower motor neuron to activate paretic or paralytic muscle. Clinical application of NMES is to improve functional activity and for therapy. One of the therapeutic effects is 'motor learning', which

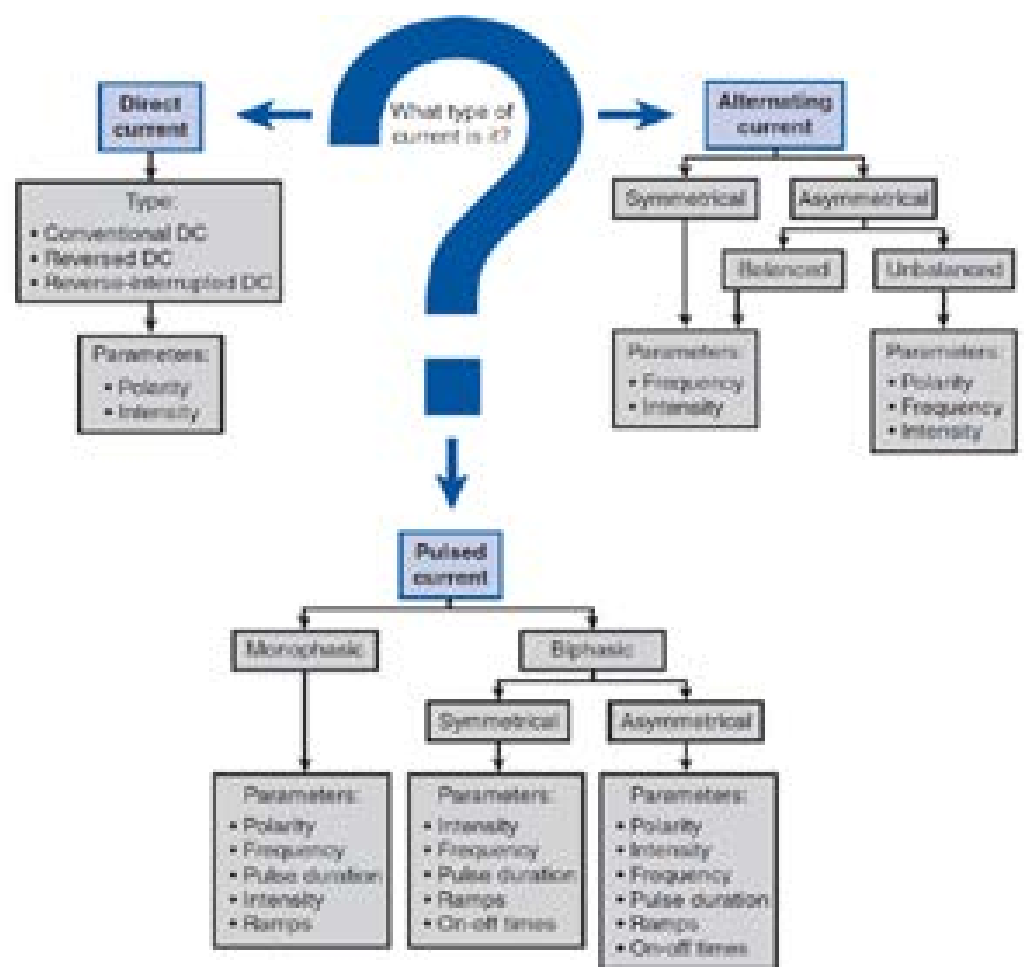

Figure 1: Classification of electrical current ${ }^{13}$ mean improving the preserved motor function which will loose due to damage of central nervous system. ${ }^{16}$

Normal muscle contraction is a response to depolarization of its motor nerve. In the absence of muscle innervation, the muscle contraction is produced by depolarization of muscle membrane, rather than the motor nerve. This stimulus will create the same muscle contraction as a natural stimulus. Once a stimulus reaches a depolarizing threshold, the nerve or muscle membrane depolarizes, and propagation of the impulse or muscle contraction occurs. ${ }^{17}$

NMES initiated with the excitation of peripheral nerve tissue. Terminology of 'stimulus threshold' is defined as the lowest level of electrical charge that causes an action potential. The threshold for action potential for nerve fibers is 100 to 1000 times less than stimulation for muscle fibers. So, the NMES will stimulate nerves directly and also the end point of the nerve proximal to neuromuscular junction..$^{16}$ The electrode placement of NMES can be done on muscle belly or motor end point. Stimulation via motor points would directly promote the motor branch excitation. Non-optimal electrode placement would require higher current levels to reach and excite the motor branch, as a consequence there will be a greater excitation of pain afferent fibers. The most effective site for electrode placement is on motor point. In muscle with more than 1 motor points, large electrodes are used for efficiency of electrical stimulation. ${ }^{15,18}$

The basic of NMES is therapeutic current. There are 3 types of therapeutic current; direct, alternating and pulsed current. The pulsed current is usually more comfortable and more commonly used in clinical practice. NMES units commonly use a symmetric biphasic pulsed current, which means they use bidirectional flow of charge and the flow of charge in each direction is the same in both pulse phases. ${ }^{15}$

Contraindications of NMES are same as all other electrical stimulation devices, e.g. pregnancy, pace maker insertion, hemophilia or 
thrombosis patients, and other relative condition such as epilepsy, seizure, and cardiac patients. ${ }^{17,19}$

\section{Nerve and Muscle Respond to NMES}

Nerve fiber recruitment in NMES is different with normal physiologic nerve recruitment. In a physiologic action potential, the recruitment starts with neurons of the smallest diameter to the largest ones. In NMES, the principle recruitment pattern is "reverse recruitment order" which mean largest

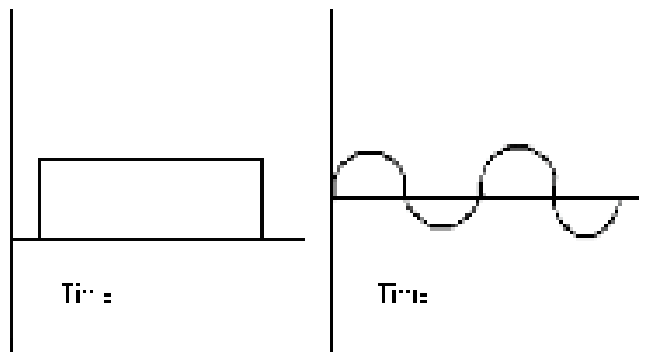

ก

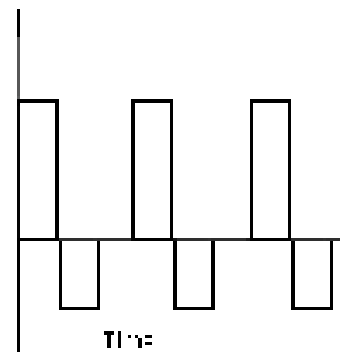

э.

Figure 2: Type of current in electrical stimulation ${ }^{14}$

as "a program of goal oriented multidisciplinary rehabilitation under the responsibility of a PMR specialist during the first days or weeks following injury, illness or response to complex medical treatment or its complications". The candidate of acute medical rehabilitation is a patient with complex disabilities that need rehabilitation service, is informed about functional prognosis of his disease or disability, or who has to live with permanent disabilities and needs possible adaptation to his environment. This information has to be conveyed to the caregiver and the family too, especially in the case of pediatric patients or severely disabled patients. Complications in patient in ICU after prolong immobilization could appear as pressure
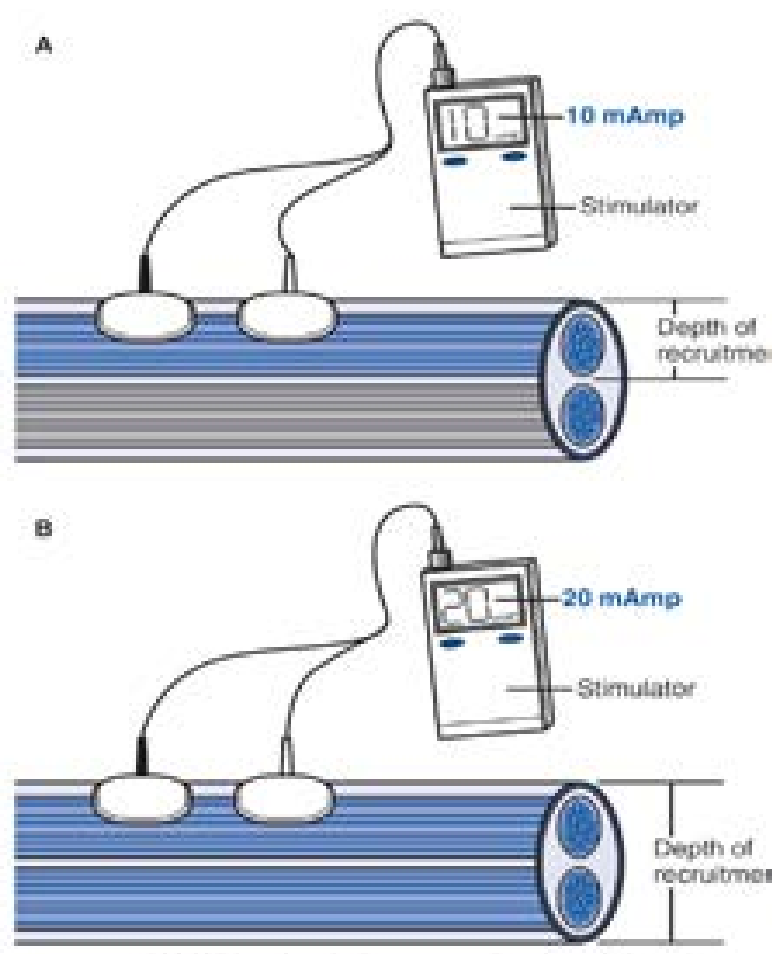

Figure 3: Higher amplitude (B) recruits more muscle fibers compared to the lower amplitude (A) ${ }^{13}$ 
ulcers, skeletal stiffness or contracture, respiratory problems, bladder and bowel problems or muscle weakness. ${ }^{21}$

\section{NMES for ICU-AW}

Muscle weakness as a consequence of prolonged immobilization could limit physical and function ability to perform routine daily activities leading to reduced quality of life. A study of NMES on lower extremities (rectus femoris and peroneus longus) in addition to standard physical rehabilitation program shows significant benefit to critically ill patient following ICU discharge. NMES and physical rehabilitation programs also enhance muscle strength (MRC muscle strength score and hand grip strength) in ICU survivors at hospital discharge. Further studies are needed on larger sample size to document improvement in these patients and long term effects. ${ }^{22}$

The feasibility and safety of NMES on quadriceps femoris in acute critically ill patient with the study outcome were to assess muscle contraction quality, identify factors that interfere contraction and the effect of NMES on cardio-respiratory system and skin. The study results showed that muscle contraction could prevent muscle weakness if stimulated with 3-4 sessions consisting of 3-4 contractions per session with an intensity of 65-69 mA. Sepsis, edema, and vasopressors after admission to medical ICU, were the factors that could impede muscle contraction that need to be stimulated with NMES. The study also concluded that NMES is safe when used in ICU patients. ${ }^{23}$

The effects of NMES use at cellular level were studied with the purpose to prove that NMES session would increase count of Endothelial Progenitor Cells (EPCs) by mobilizing progenitor endothelial cells in critically ill septic patients. The NMES electrodes were placed on the motor points of vastus lateralis, vastus medialis and peroneus longus in both of the legs, the intensity using high and medium frequency protocol increasing after every 3 minutes. The results of blood sampling and flow cytometry analyses show that NMES mobilized EPCs in acutely ill; however, it was not dependent on NMES protocol, rather more on corticosteroid administration. ${ }^{24}$

Benefits of using NMES in patient receiving mechanical ventilation have also been studied with an aim to prove benefit of early rehabilitation and NMES in this group of patients. The study placed NMES in quadriceps, tibialis and gastrocnemius in both lower extremities for 60 min daily until visible contraction was detected. There were alternating phases of contractions and rest phase during NMES stimulation. It is novel to use NMES earlier in critical care, because it can preserve the muscle strength. The result of this study stressed the need of more multicenter randomized trials in phase II of critical illness to confirm benefits of NMES. ${ }^{25}$

Systematic review study of NMES in critical care using International Classification of Functioning, Disability and Health (ICF) was aimed to evaluate the evidence of NMES efficacy compared with usual care in critical care and its impact on all domains in ICF. Limitations of the study were that the study included only a small number of eligible patients and there was heterogeneity in outcome, but the researcher concluded that NMES increased muscle strength and could be beneficial in increasing range of movements in joints and prevention of muscle atrophy during mechanical ventilation and activity limitation.

Conflict of interest: None declared by the authors

Authors' contribution: All authors took equal part in concept, literature search, manuscript writing and editing of this manuscript 


\section{REFERENCES}

1. Gosselink R, Needham D, Hermans G. ICU-based rehabilitation and its appropriate metrics. 2012:533539. [PubMed] doi:10.1097/ MCC.0b013e328357f022

2. Vrbová G, Hudlicka O, Centofanti KS Application of Muscle/Nerve Stimulation in Health and Disease.; 2008.

3. Batt J, Herridge M, dos Santos C. Mechanism of ICU-acquired weakness: skeletal muscle loss in critical illness. Intensive Care Med. 2017;43(12):1844-1846. [PubMed] doi:10.1007/s00134-017-4758-4

4. Koo KK, Fan E. ICU-Acquired Weakness and Early Rehabilitation in the Critically III. JCOM. 2013;2(5):223231.

5. Richard A, John K. Intensive care unit-acquired weakness. Contin Educ Anaesthesia, Crit Care Pain. 2012;12(2):62-66. [Free full text] doi:10.1093/bjaceaccp/mkr057

6. Khoiny AS, Behrouz R. Critical Illness Polyneuropathy and Critical Illness Myopathy. Pract Neurol. 2011;(June 2011):52-56. [Free full text]

7. Hermans G, Berghe G Van Den. Clinical review : intensive care unit acquired weakness. Crit Care. 2015:1-9. [PubMed] [Free full text] doi:10.1186/ s13054-015-0993-7

8. Intiso D. ICU-acquired weakness: Should medical sovereignty belong to any specialist? Crit Care. 2018;22(1):1-5. [PubMed] [Free full text] doi:10.1186/s13054-017-19237

9. Deem S. Intensive-Care-Unit-Acquired Muscle Weakness Introduction Definitions Incidence Pathology of ICUAcquired Weakness Risk Factors and Pathogenesis Clinical Presentation and Outcomes Diagnosis Prevention and Therapy. Respir Care. 2006;51(9):1042-1052 [PubMed]

10. Kress JP, Hall JB. ICU-Acquired Weakness and Recovery from Critical Illness. N Engl J Med.
2014;370(17):1626-1635. [PubMed] doi:10.1056/NEJMra1209390

11. De Jonghe B. Paresis Acquired in the Intensive Care Unit: A Prospective Multicenter Study. JAMA. 2002;288(22):2859. [PubMed] doi:10.1001/jama.288.22.2859

12. Chae, Triolo, Kilgore, Creasey, DiMarco. Functional Neuromuscular StimuIation. In: Delisa J, Walsh N, editors. Physical Medicine and Rehabilitation Principles and Practice $4^{\text {th }}$ edition. Philadelphia: Lippincott Williams and Wilkins; 2005. p.1405-26.

13. Bellew. Foundation of Electrotherapy. In: Wolf SL, editor. Modalities for Therapeutic Intervention $5^{\text {th }}$ edition. Philadelphia: FA Davis Company; 2012. p. 209-38.

14. Shapiro. Electrical Currents. In: Cameron $\mathrm{MH}$, editor. Physical Agents in Rehabilitation from Research to Practice $2^{\text {nd }}$ edition. St Louis: Saunders; 2003. p. 19-54.

15. Lake DA. Neuromuscular Electrical Stimulation: An Overview and its Application in the Treatment of Sports Injuries. Sports Medicine; 1992:13(5):320-336. [PubMed]

16. Sheffler LR, Chae J. Neuromuscular electrical stimulation in neurorehabilitation. Muscle Nerve. 2007;35(5):562-590. [PubMed] doi:10.1002/mus.20758.

17. Hooker DN and Prentice WE. Basic Principles of Electricity and Electrical Stimulating Currents. In: Prentice WE, editor. Therapeutic Modalities: for Sports Medicine and Athletic Training Sixth Edition. New York: Mc Graw Hill Companies; 2009: p. 104-149.

18. Hainaut K, Duchateau J. Neuromuscular Electrical Stimulation and Voluntary Exercise. Sports Medicine. 1992;14 (2): 100-113. [PubMed]

19. Knutson, Scheffler \& Chae. Functional Neuromuscular Electrical Stimulation. In: Frontera WR (ed.) DeLisa's Physical Medicine and Rehabilitation. 5th ed. Philadelphia: Lippincott Williams and Wilkins; 2010: p. 1977-92.

20. Cristian A, Green J. Patient safety and Quality Improvement in Rehabilitation Medicine. In: Cristian A and Carter GT, editors. Patient Safety in Rehabilitation Medicine: Physical Medicine and Rehabilitation Clinics of North America. Vol 23. USA: WB Saunders Company; 2012: p. 221-30

21. Stam HJ, Buyruk HM, Melvin JL, Stucki G. Introduction to Acute Medical Rehabilitation. In: Stam \& Buyruk, editors. Acute Medical Rehabilitation Vol I. Turkey: Odak Ofset Ankara; 2012: p. 13-18

22. Patsaki I, Gerovasili V, Sidiras G, Karatzanos E, Mitsiou G, Papadopoulos E, et al. Effect of neuromuscular stimulation and individual rehabilitation on muscle strength in Intensive Care Unit survivors; A randomized trial. J Crit Care 2017;40:76-82 [PubMed] DOI: 10.1016/j.jcrc.2017.03.014

23. Segers J, Hermans G, Bruyninckx F, Meyfroidt G, Langer D, Gosselink R. Feasibility of neuromuscular electrical stimulation in critically ill patients. J Crit Care 2014;29:1082-88 [PubMed] doi: 10.1016/j.jcrc.2014.06.024

24. Stefanou C, Karatzanous E, Mitsiou G, Psarra K, Angelopoulos E, Dimopoulos S, et al. Neuromuscular electrical stimulation acutely mobilizes endothelial progenitor cells in critically ill patients with sepsis. Ann Intensive Care 2016; 6:21 [PubMed] [Free full text] doi: 10.1186/s13613-0160123-y.

25. Kho ME, Truong AD, Brower RG, Palmer JB, Fan E, Zanni JM, et al. Neuromuscular Electrical Stimulation for Intensive Care Unit-Acquired Weakness: Protocol and Methodological Implications for a Randomized, Sham-Controlled, Phase II Trial. Phys Ther. 2012;92(12):1564-1579. doi:10.2522/ptj.20110437 [PubMed] [Free full text]

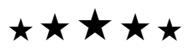

